\title{
LOS ESTUDIOS PSICOLÓGICOS DEL ESTABLECIMIENTO DE AGENDA: ESPECIRCACIÓN Y DISCUSIÓN DE UN MODELO DE IDENTIDAD CONFORMISTA VERSUS COOPERATIVISTA
}

\author{
Cruz Garc ía Linios \\ Universidad Autónoma del Estado de México
}

http://dx.doi.org/10.5209/NOMA.53341

\begin{abstract}
Resumen.- Antecedentes. Los estudios psicológicos del establecimiento de agenda han demostrado que; 1) la propaganda del Estado incide sobre la identidad ciudadana mediante fuentes, mensajes, audiencias y contextos que; 2) legitiman la rectoría del Estado siempre que los receptores consideren que la información está argumentada y sustentada en evidencias, así como; 3) su repercusión en altos y bajos niveles de elaboración de pensamientos, emocionales, deliberados, planificados y/o sistemáticos los cuales demuestran; 4) reconocimiento de méritos y la confianza de la sociedad para con sus autoridades que a su vez generarán; 5) identidades conformistas más persistentes conforme transcurre el tiempo o se contrasten con eventos de lesa humanidad que cuestionen la rectoría del Estado y su propaganda ocasionando incluso; 6) una defensa del régimen político o la forma de gobierno en materia de seguridad pública.
\end{abstract}

Objetivo. Especificar un modelo para el estudio de la propaganda, agenda e identidad sociopolítica que explique las relaciones complejas entre sociedad y Estado en materia de seguridad y pacificación.

Método. Se llevó a cabo un estudio no experimental, documental retrospectivo y exploratorio con una selección no probabilística de artículos indexados en repositorios de LATINDEX, REDALYC y DIALNET considerando el criterio de reciente publicación 2010 a 2016.

Resultados. Se especificó un modelo para el estudio de la propaganda, agenda e identidad sociopolítica entre el estado y la ciudadanía en materia de seguridad, violencia, pacificación, conformismo y cooperativismo.

Discusión. El modelo específicado sólo explica dos procesos alusivos al estudio de la propaganda, agenda e identidad sociopolítica sin considerar otras identidades apolíticas o subpolíticas tales como la indefensión, la indolencia y la indiferencia atribuida a los sectores jóvenes de la sociedad civil.

Conclusión. Es menester revisar e incluir en el modelo variables que expliquen la indefensión, indolencia e indiferencia de sectores jóvenes de la sociedad civil en escenarios digitales como Facebook, Twitter, Instagram, Periscope y Whatsapp a fin de poder anticipar escenarios de genocidio o indiferencia.

Palabrasclave:seguridad, agenda, identidad, conformismo, coopera tivismo.

\section{Psychologic alstudies of agenda: Specification and disc ussion of a model of cooperative versus conformistidentity}


Abstract- Background. Psychologicalstudies of agenda settinghaveshownthat; 1) state propaganda impactoncitizenidentitythroughsources, messages, audiences and contexts; 2) legitimizingtheguidance of thestateprovidedthe receivers considerthattheinformationisreasoned and supportedbyevidence, and; 3) itsimpactonhigh and lowlevels of development of thoughts, emotional, deliberate, planned and / orwhich show systematic; 4) recognition of merit and confidence of society to theirauthoritieswhich, in turngenerate; 5) more conformistidentitiesthatpersistent as time orcontrastwitheventsagainsthumanitythatquestiontheguidance of thestate and propaganda causingeven; 6) a defense of thepoliticalregimeorform of governmentonpublicsecurity.

Objective. Specify a modelforthestudy of propaganda, sociopolitical agenda and identitythatexplainsthecomplexrelationshipsbetweensociety and statesecurity and peace.

Method. a non-experimental study, retrospectivedocumentary and exploratorywith a nonrandomselection of articlesindexedrepositories LATINDEX, REDALYC and DIALNET wascarriedoutconsideringthecriterionrecentlypublished 2010-2016.

Results. amodelforthestudy of propaganda, sociopolitical agenda and identitybetweenstate and citizensecurity, violence, peace, conformism and cooperativismspecific.

Discussion. Specifiedmodelonlyexplainstwoallusiveprocesses to thestudy of propaganda, sociopolitical agenda and identitywithoutconsideringother nonpoliticalidentitiesor sub-policiessuch as helplessness, indolence and indifferenceattributed to theyouthsectors of civil society.

Conclusion. Itisnecessary to review and include in themodel variables thatexplaintheindefension, indolence and indifference of youthsectors of civil society in digital scenariossuch as Facebook, Twitter, Instagram, WhatsApp Periscope and in order to anticipatescenarios of genocideorindifference.

Keywords;sec unity a genda identity, conformism, cooperativism.

El objetivo del presente trabajo es describir, especificar y discutir los ejes teóricos, conceptuales y empíricos en torno al establecimiento de una agenda sociopolítica -difusión sistemática de la seguridad pública, la rectoría del Estado y la desconfianza ciudadana hacia sus gobernantes-, así como su incidencia sobre la identidad civil ante la delincuencia, violencia, corrupción, negligencia, opacidad o nepotismo de los gobernantes con respecto a sus gobernados a fin de poder anticipar escenarios de conflicto y cambio social. 
¿Cuáles son las dimensiones, las trayectorias de las relaciones de dependencia, los alcances y límites de la seguridad pública, el establecimiento de agenda y la identidad sociopolítica que se observan en la propaganda conformista -el Estado como rector de la seguridad, procurador del delito e impartidor de justicia, así como la sociedad confinada al auto-cuidado- más que la propaganda cooperativista -la sociedad y el Estado corresponsables ante la construcción de la paz pública y estilos de vida libres de violencia-?

La hipótesis de trabajo que responde a tal cuestionamiento plantea; 1) la transformación del Estado autoritario cuyas instituciones de seguridad son coercitivas, pero legitimadas por una propaganda conformista la cual, sin embargo; 2) evolucionó hasta convertirse en una agenda establecida en los medios de comunicación para resaltar la rectoría del Estado en materia de seguridad, aunque; 3) los sectores civiles organizados en esferas y redes generaron un sistema de contrapropaganda indicada por la construcción de una paz social mediante la adopción de estilos de vida y discursos libres de violencia.

En este sentido, el propósito del escrito consiste en especificar un modelo para el estudio de la problemática a fin de poder anticipar escenarios de conflictos por la administración de los recursos naturales y los servicios públicos.

El análisis de los datos, teorías, conceptos y hallazgos se realizó a partir de una investigación documental de julio 2010 a noviembre 2013 en las bases de datos de DIALNET, LATINDEX y REDALYC por considerarlas líderes en América Latina. La búsqueda de información se llevó a cabo a partir de las combinaciones de palabras claves tales como; Estado, ciudadanía, violencia, delincuencia, seguridad, percepción y riesgo. Se seleccionaron aquellos artículos con resultados empíricos preferentemente de la región y localidad. Posteriormente, se realizó un análisis de contenido para establecer los ejes y temas de la agenda investigativa. La información fue sistematizada siguiendo la técnica de síntomas para el desarrollo de modelos. En relación con el estado del conocimiento, la propuesta del modelo fue contrastada con los hallazgos empíricos.

\section{Seguridad pública}

Los conceptos de seguridad e identidad son temas centrales en la agenda global, regional, política, democrática, social, pública, urbana, 
ciudadana (Carcelén, Esteba y Peyró, 2013). Se trata de una pieza clave en el ajedrez de la geopolítica y los tratados bilaterales de corresponsabilidad; las estrategias de beligerancia y los movimientos de reivindicación; las políticas territoriales y la apropiación del espacio; la contienda política y la participación electoral; los programas de combate a la delincuencia y la acción colectiva de grupos vulnerables (Guardiola, Espinar y Hernández, 2010). En este sentido, la relación entre seguridad e identidad es bidireccional ya que la construcción de una agenda, en materia de seguridad implica el consenso de identidades y la formación de una ciudadanía requiere de programas de seguridad que garanticen los derechos humanos. Por ello, la acción y decisión gubernamental están ligadas con la participación social (Izquierdo, 2012).

En el marco de los tratados internacionales, los Estados construyen acuerdos para eficientar la corresponsabilidad en materia de seguridad. Respecto al rubro de seguridad regional ésta es entendida como un instrumento de cooperación técnica entre países que comparten un bloque económico-territorial. De este modo, la seguridad democrática y regional son instrumentos de poder que suponen el fortalecimiento de una esfera civil internacional desde la cual se vigila la acción gubernamental, se complementan las decisiones públicas y se enriquecen los programas de atención a víctimas. Es posible observar que la emergencia de una esfera civil internacional requiere del acuerdo entre países, pero supone la expansión de principios rectores de la ciudadanía frente a los fenómenos de inseguridad que atentan contra la estabilidad y el equilibrio de poder entre el Estado y la ciudadanía. Se trata de una identidad civil en la que se discuten los temas de la agenda pública, la seguridad nacional entre ellos (Wasike, 2013).

A pesar de que las fronteras cada vez son más tenues entre las naciones, los estados al mismo tiempo que facilitan las libertades ciudadanas que le permiten manifestar sus ideas en otras latitudes, incentivan una identidad nacional a partir de políticas consolidadas en cuanto a limitación de inmigrantes, restricción de inversiones en telecomunicaciones o selección de prospectos laborales. Se trata de un programa de construcción o reconstrucción según la historia de cada país de una identidad nacional. En este enfoque, la seguridad nacional alude a principios vigía que orientan las decisiones políticas y encamina las acciones ciudadanas a la búsqueda de la defensa de lo propio y la 
exacerbación de lo extraño ya no como un riesgo, sino como un grupo de referencia para la competitividad, el crecimiento o el desarrollo. A pesar de ello, los grupos radicales de la sociedad, lejos de participar en la construcción de una esfera civil, intensifican sus acciones de riesgo y ponen en peligro la democracia, la región o la nación misma. Por ello, se propone la seguridad social en la que su principal fundamento es el pacto civil (Zaffaroni, 2011).

El concepto de seguridad pública supone el establecimiento de la paz pública a partir de la emergencia de la prevención del delito y la administración punitiva. También denota un orden social a partir de su restablecimiento por sistematización de diagnósticos y procesos que reduzcan los efectos del crimen organizado. Alude a un proceso quirúrgico en el que la defensa del territorio se traduzca en confianza hacia las autoridades (Añez, Rujano y Párraga, 2011). En otro sentido espacial, implica la creación de los escenarios que propicien asambleas comunitarias, así como el marco de discusión y acción a seguir ante las problemáticas que afectan la paz pública (Salas, 2011). Todos los enfoques, social, espacial y simbólico apuntan a una situación deseable o externa en referencia a un proceso indeseable o interno (Calderón, 2012). Por ello, el concepto de seguridad pública está anclado al pasado en referencia al futuro. Se trata de percepciones de aversión y propensión en clara alusión a un antes y después de la confianza o desconfianza por parte de la ciudadanía hacia el Estado (Ulloa, 2012). En dicho proceso, la institución pretoriana es protagonista ya que la recuperación de la confianza ciudadana o el descrédito hacia la misma está centrada en las fuerzas del orden (Zúñiga, 2012).

No obstante, un análisis minucioso del concepto violencia parece mostrar que ésta no sólo es un indicador de inseguridad, sino también de identidad. El concepto violencia atraviesa ambos términos ya que puede ser producto de la ausencia de reconocimiento social, la omnipotencia del Estado, la exclusión social y las asimetrías entre la clase política y demás clases sociales (Acosta, 2010). Es decir, la violencia alude a entornos públicos y privados en los que el conflicto es generado por ausencia de acuerdos, pero también por valores inherentes a la naturaleza humana. La cuestión aquí es fundamental: la seguridad y la identidad, ambas son partes fundamentales de la construcción de una esfera civil ya que la inseguridad y la violencia, principales amenazas de su gestación, están presentes en las dimensiones públicas y privadas, macro y micro social (Rodríguez, 2010). 
Sólo resta analizar una consecuencia de la ausencia de esfera civil: la privacidad. Establecida como un conjunto de derechos y obligaciones, la privacidad alude a facultades, libertades, garantías, singularidad, autonomía y capacidades frente a sistemas informativos espías que atentan contra la invisibilidad voluntaria de un usuario de tecnologías de información y comunicación (García, 2010).

Las sociedades según el estado del arte conceptual, parecen avanzar hacia escenarios de información y comunicación que trastocan su poder de elección y decisión en acciones exploratorias de espacios, inseguridad, violencia o privacidad, pero dependiente de los avances y las innovaciones tecnológicas, la ciudadanía trata de construir una esfera civil que la pueda proteger del crimen y la delincuencia (Latorre, 2011).

Sin embargo, los medios de comunicación resultan ser interventores directos y colaterales de las percepciones de inseguridad pública al momento de evaluar las estrategias gubernamentales y la acción policiaca contra la delincuencia (García, 2011).

La naturaleza esta mediatizada por diversos contextos significativamente diferentes. La naturaleza puede ser representada como contextos de salud o turismo, ciencia, academia, política, agricultura, derecho, industria o comercio, concienciación, gestión o contaminación, desastres, radioactividad y enfermedad, nacionalismo, naturalismo, expansionismo, catastrofismo, sostenibilismo, territorialismo, economismo y legalismo, nacionalismo, regionalismo, localismo, antrópismo, abiótismo, biotismo, clima, energía y agua (González, Pombo, Méndez, Espejel y Leyva, 2011).

Si los estudios del establecimiento de agenda se avocan a explicar las relaciones existentes entre los contenidos de los medios de comunicación, los temas de opinión pública y los rubros de las políticas públicas, los estudios del efecto encuadre advierten que el estilo de encuadre y penetración mediática incide directamente en la socialización de la ciudadanía (GuyGoldfarb, 2010).

Se trata de un círculo vicioso o virtuoso en el que la difusión de los hechos es un indicador de ingobernabilidad o democracia que retroalimenta los temas de discusión de la agenda ciudadana. En referencia a la amplitud de los medios de comunicación, los regímenes autoritarios parecen construir relaciones de desconfianza y las 
democracias parecen construir relaciones de confianza entre los ciudadanos, los medios y la res política (Orozcoy Franco, 2012).

\section{Establecimiento de agenda}

Groshek (2011) encontró relaciones positivas y significativas entre tres medios de comunicación (televisión, radio y prensa) con respecto a la situación sociopolítica de 122 países. A medida que el encuadre y penetración de los medios se intensificaba, contribuía con el aumento de prácticas democráticas. Las diferencias existentes entre la televisión, radio y prensa corroboraron el supuesto en torno al cual los medios de comunicación contribuyen en la construcción de una democracia participativa.

Sin embargo, Wirth, Matthes, Schemer, Wettstein, Friemel, HânggliySiegert (2010) llevaron a cabo un estudio en el que correlacionaron la prominencia de los argumentos mediáticos, públicos y políticos. Establecieron asociaciones positivas entre los argumentos públicos y políticos con los argumentos mediáticos en tres niveles de amplitud; baja, mediana y alta. Al comparar los discursos mediáticos de alta y baja influencia, los autores encontraron que las asociaciones fueron significativas en un solo nivel de amplitud intermedio, ni muy alto ni muy bajo.

A diferencia de los estudios del efecto agenda, los estudios del efecto encuadre observan que el estilo de comunicación incide más que la amplitud del medio. A partir del supuesto según el cual el establecimiento de la agenda estaría indicado por efectos de encuadre más que por la correlación entre los contenidos mediáticos y los temas de opinión pública, García (2014) llevó a cabo un trabajo de sistematización de los encuadres noticiosos y encontró una relación positiva con los temas de opinión pública. En el trabajo de Bizer, LarsenyPetty (2010) el efecto de encuadre tuvo una relación indirecta con la intención. A través de la certeza de la fuente, los estilos de encuadre y difusión incidieron sobre las decisiones de los individuos. Al comparar la relación directa con la indirecta, el efecto de encuadre parece haber sido incrementado por la mediación de la credibilidad de la fuente.

Antes bien, la percepción ciudadana en torno a los contenidos de la prensa parece estar más próxima a la desconfianza. Flores y Mendieta (2012) encontraron mayores porcentajes en la percepción negativa de 
la prensa. En su mayoría, los lectores consideran que los contenidos incitan a la violencia. En este estudio, la activación de emociones tales como, enojo, tristeza, desagrado e inseguridad podrían estar relacionados con acontecimientos difundidos por otros medios de comunicación que al ser asociados con las notas de prensa complementarían un ciclo de persuasión en el que los medios de comunicación masiva estarían avocados a mostrar escenarios disuasivos de la paz pública. El trabajo de Campillo (2012) muestra que la difusión de la seguridad pública es proporcionalmente neutra y supera el sesgo a favor o en contra en la muestra revisada de 1995 a 2007, aunque el encuadre de la prensa es considerado como fortuita por la ciudadanía respecto al tema de la inseguridad. Tal hallazgo, corrobora la hipótesis en torno a la cual la incidencia de los medios impresos parece ser explicada por un procesamiento automático de la información más que por un proceso deliberado, planificado y sistemático.

A partir de que la ciudadanía percibe a los medios de comunicación como emisores de contenidos nocivos para la democracia, la equidad y la paz pública, los estudios del efecto intensidad se han centrado a establecer la relación existente entre los mensajes y los receptores. Nisbet, StoycheffyPearce (2012) establecieron el efecto directo del uso de internet (género, escolaridad y residencia) sobre la demanda ciudadana de democracia. A medida que los hombres, tienen un mayor nivel académico y han permanecido en la localidad, incrementan sus demandas de información relacionada con la democracia. Por su parte Humanes y Moreno (2012) encontraron que el sexo repercutía en el consumo de noticias relativas a la inmigración mientras que la ideología repercutía en las noticias alusivas al terrorismo.

En síntesis, los estudios del efecto intensidad parecen demostrar que los contenidos periodísticos al estar focalizados en las problemáticas locales, activarían emociones que corresponderían con decisiones y acciones imprevistas en sus lectores. El sesgo de los rotativos en su encuadre de los hechos y las diferencias entre los diarios locales, parecen evidenciar un entramado de noticias sin restricción que a pesar de su sistemática difusión tendrían efectos diferenciados en los receptores.

En consideración a otros trabajos realizados por Carreón (2014) y García (2013) en los que los medios de comunicación son los propulsores de la delincuencia como producto de las falas del sistema sociopolítico y la 
ineficacia de funcionarios encargados de velar por la paz pública. en el presente trabajo, los medios de comunicación son actores fundamentales para explicar la construcción de una identidad sociopolítica en la que la inseguridad y violencia son temas desde los que se cuestiona al estado como rector de la vida nacional.

Ahora bien, en referencia a la Teoría de la Política Criminológica (TPC) en la que se estipula el control delictivo por parte del Estado y cuyo impacto en la ciudadanía es la percepción de seguridad (Fondevilla y Quintana, 2013), en el presente trabajo hemos establecido que la identidad sociopolítica es más bien resultado de la influencia de los discursos, ya no presidenciales o institucionales como lo advierte la TPC, sino desde los discursos de la sociedad en referencia al accionar de sus gobernantes y fuerzas del orden público.

\section{Identidad sociopolítica}

La identidad sociopolítica entendida como creencias, percepciones, actitudes, decisiones y acciones relativas a la evaluación de políticas públicas y programas emanados del Estado es un constructo que explica la relación entre ciudadanía y gobierno.

En el marco de la seguridad pública, la ciudadanía desarrolla percepciones de inseguridad que suponen la emergencia de una identidad sociopolítica. Indicada por grados de miedo, incertidumbre, enojo o indignación, la sociedad civil produce emociones hacia sus gobernantes que los llevan a un estado de indefensión. Se trata de un proceso de desconfianza en el que los gobernantes y gobernados están inmersos.

Los delitos del fuero local y los delitos del fuero federal están concentrados en la capital del país, pero la densidad poblacional no sólo explica el aumento de los casos, sino además la formación de grupos, la emergencia de conflictos y el afloramiento de cambios sociales. Es decir, la identidad sociopolítica se caracterizaría, de acuerdo con los datos de INEGI (2010) como un emprendedurismo de seguridad que los ciudadanos desarrollaron a partir de la impericia del Estado.

Definida como imperativo territorial, económico, político, social, institucional y grupal, la identidad es un factor explicativo de las decisiones y acciones del individuo que se inmiscuye en los asuntos públicos; discute los temas de la agenda social; interpreta el marco 
jurídico regulatorio; organiza manifestaciones colectivas o virtuales (Borghello y Temperi, 2012).

Si cada una de las dimensiones de la identidad, según el estado del arte, es relacionada con las dimensiones de la seguridad pública y ciudadana, entonces tenemos una matriz que va de la exploración de instituciones al compromiso con las mismas. En un extremo, la ciudadanía desconfía de sí misma y de las agencias gubernamentales. En el otro extremo, la confianza en las instituciones es el indicador de la discusión y acción social. En medio, el uso de tecnologías de información y comunicación permite el debate virtual de la agenda ciudadana, pero la hace invisible, incluso la transmuta en roles volátiles y efímeros.

A medida que la exploración se intensifica, los roles se diversifican y el compromiso social se hace difuso. En este escenario, la construcción de la esfera civil se ha estancado, aunque se reactiva cuando la inseguridad es percibida como lejana o cercana. En el primer, caso la ciudadanía transita de lo privado a lo público y en el segundo, materializa la información circundante en acciones concretas de protección civil. Tal proceso supone el encuadre de los medios de comunicación (García, 2012). Es decir, la información relativa a la seguridad es seleccionada de acuerdo con el perfil de las audiencias, la intensificación de los mensajes, editoriales, reportajes o columnas de la televisión, radio y prensa influyen en la opinión pública para infiltrar temas en la agenda pública (Mao, Richter, Burns y Chaw, 2012). El abatimiento del encuadre periodístico sólo sería posible con la construcción de una opinión pública por parte de la esfera civil, pero ésta también es influida por los comunicadores. En efecto, la construcción de una esfera civil protectora de la inseguridad y la negligencia $u$ opacidad del Estado, se gesta desde los medios de comunicación.

El aporte del estado del conocimiento consiste en documentar y contrastar la información relativa a la identidad sociopolítica como un fenómeno resultante de las asimetrías entre sociedad y Estado. La identidad sociopolítica, en el contexto de la seguridad pública, supone un conjunto de representaciones que legitiman la rectoría del Estado en materia de control delictivo, pero una vez que la ciudadanía percibe que la corrupción, negligencia, nepotismo $u$ opacidad de sus autoridades están vinculadas con la delincuencia organizada, desarrolla percepciones de inseguridad en las que el poder político se 
difumina y la influencia social se disemina para reproducir la dominación, el control, indefensión y conformismo social.

En este panorama de poder jurídico-político e influencia sociopolítica, la relación entre gobernantes y gobernados se torna difusa, ya que la sociedad parece estar en una disyuntiva de legitimar la rectoría del gobierno que incluiría medidas de combate a la delincuencia frente a la seguridad privada que supone la inversión de grupos radicales en servicios de video vigilancia. Es decir, los escenarios que se avizoran parecen orientarse desde la reducción de la participación institucional y el exacerbamiento de grupos civiles que tendrían facultades para vigilar, denunciar, capturar, procesar y enjuiciar a delincuentes según criterios de seguridad privada y defensa del patrimonio más que de rehabilitación social, o bien, escenarios en los que las instituciones de seguridad son administradas por la iniciativa privada bajo el imperativo de la minimización de costos y maximización de ganancias.

En cualquiera de estos escenarios la identidad sociopolítica estaría fragmentada en intereses particulares o grupales que no sólo procuran la defensa del patrimonio o la integridad personal, sino el cumplimiento de convicciones o prejuicios que fragmentarían la paz pública.

La identidad sociopolítica, a diferencia de la rectoría del Estado, está llamada a explicar los escenarios posibles de seguridad e inseguridad, pero sobre todo a ser un tema central en la agenda pública. En este sentido, el papel de los medios de comunicación es fundamental ya que su encuadre no sólo construye una opinión ciudadana, sino además podría incidir en sus acciones de seguridad privada o en las evaluaciones de la seguridad desregulada del Estado y administrada por actores privados.

\section{Especific ación del modelo}

A partir de la revisión de la literatura concerniente a la seguridad pública, el establecimiento de agenda y la identidad sociopolítica, es posible advertir dos procesos simultáneos entra la sociedad y el Estado (ver Figura 1). 


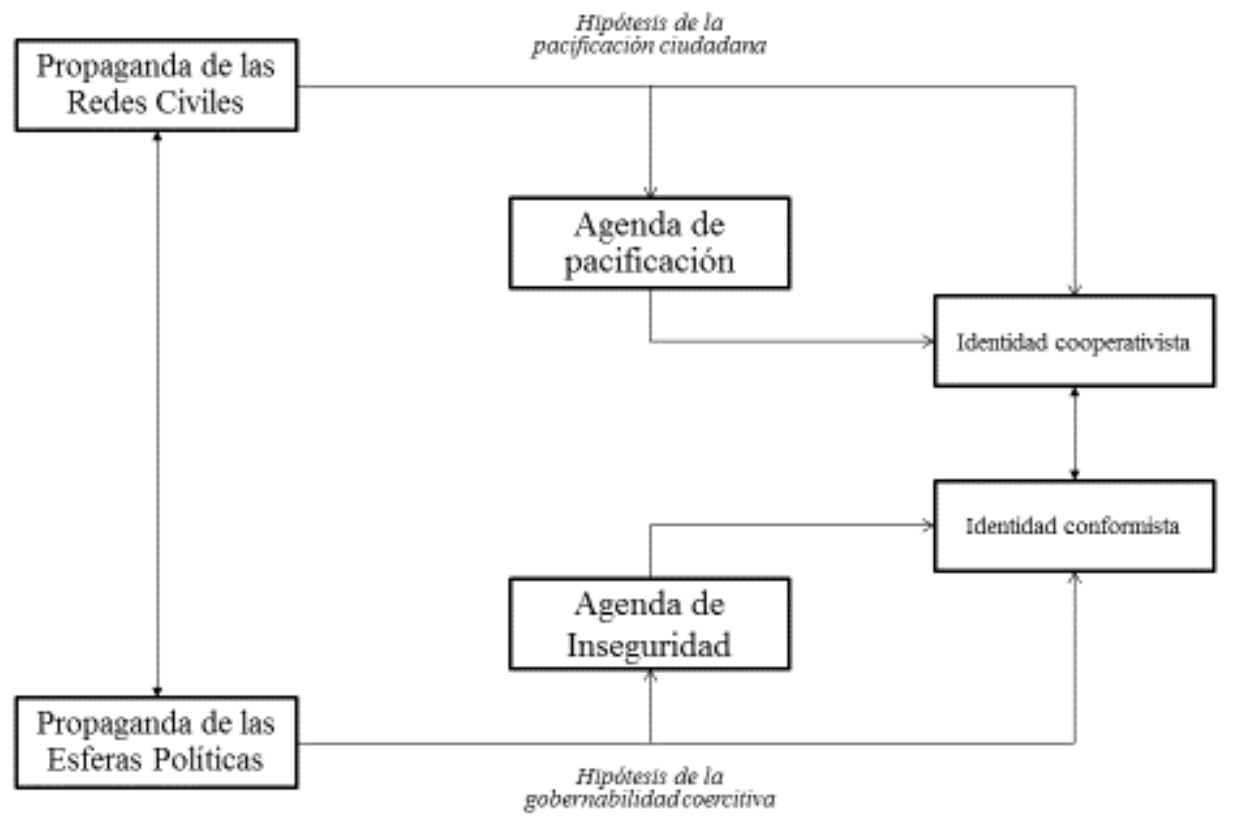

Figura 1. Especificación del modelo de relaciones de dependencia entre las variables polític as, mediáticas y sociopolític as

Hipótesis de la gobemabilidad coercitiva.

Propaganda de las esferas políticas $\rightarrow$ agenda de inseguridad identidad conformista. La rectoría del Estado en materia de seguridad, se traduce en la difusión sistemática de información relativa a la conservación de un territorio; la gestión y administración de instituciones avocadas a la prevención del delito y la procuración de justicia; así como al establecimiento de temas concernientes a la impunidad, opacidad, negligencia y corrupción; por consiguiente, la reproducción de una identidad defensora de los usos y costumbres, valores y normas tradicionales que legitiman la coerción a fin de erradicar la delincuencia.

Sin embargo, la hipótesis dela gobernabilidad coercitiva no explica la organización de la sociedad civil en esferas y redes de conocimiento encaminadas a la construcción de la paz social.

Hipótesis de la pacific ación ciudadana.

Propaganda de las redes civiles $\rightarrow$ agenda de pacificación identidad cooperativista. Del mismo modo que la propaganda del Estado se disemina en los medios de comunicación -televisión, radio, prensa, cine, Internet, telefonía fija y móvil-, la propaganda de la ciudadanía se difunde se persona a persona. Se trata de un sistema 
comunicativo en el que prevalece el discurso de la confianza hasta la corresponsabilidad.

La agenda de pacificación, a diferencia de la agenda de inseguridad, consiste en el establecimiento de temas relativos a la confianza, el compromiso, el emprendimiento, la innovación, la satisfacción, la deliberación, el consenso y la corresponsabilidad. En cada temática la sociedad civil se estructura en grupos de discusión con la finalidad de producir mensajes alusivos a propuestas e iniciativas que generen el cambio social.

La identidad cooperativista, en relación con el conformismo, supone una serie de estrategias tales como el trueque, la reutilización o el reciclaje de ideas $u$ objetos para establecer una agenda adecuada a grupos y sectores civiles que establecen la deliberación, los acuerdos y la corresponsabilidad.

Se trata de redes de conocimiento que difunden sistemáticamente discursos y estilos de vida alusivos al diálogo de saberes, el reconocimiento de diferencias y el establecimiento de programas y estrategias que equilibren las asimetrías entre los sectores civiles con respecto a las clases políticas.

\section{Disc usión y Conclusión}

Los estudios psicológicos del establecimiento de agenda con énfasis en la identidad conformista versus la identidad cooperativista se han enfocado en dos procesos; 1) el relativo al efecto de la propaganda de la rectoría del Estado sobre la identidad del miedo, la zozobra y la desconfianza de la ciudadanía con respecto a la clase política, servidores públicos, funcionarios y autoridades; 2) el relativo al efecto de la iniciativas civiles con respecto al establecimiento de temas en una agenda ética y su incidencia en una identidad observada en la cooperación.

El presente trabajo se avocó a documentar la relación existente entre ambos procesos en los que prevalece la seguridad pública, el establecimiento de agenda y la generación de una identidad sociopolítica con la finalidad de especificar un modelo para su estudio, discusión y anticipación de escenarios de inseguridad y/o pacificación.

Sin embargo, el modelo específicado para el estudio de los dos procesos sociopolíticos evidencia sólo dos trayectorias de relaciones de 
dependencia entre las categorías y las variables esgrimidas en la revisión de la literatura, pero no incluye otras rutas explicativas de la identidad apolítica o subpolítica.

En tal sentido, es menester profundizar en la revisión y modelación de variables tales como la desesperanza aprendida, el desapego e indolencia civil, la indiferencia social o el sentido de evitación de contacto que explicarían los efectos de una propaganda no difundida por el Estado o la sociedad civil sobre la identidad ciudadana.

Chávez et al., (2016) han demostrado que las actitudes hacia la muerte pueden derivarse de escenarios de duelo en el que los regímenes de gobierno y las formas de Estado producen diversas emociones que se transforman a largo plazo en indefensión.

En la medida en que los sectores jóvenes de la población se desvinculan de las clases políticas sus estilos de vida contrastan con la identidad de adultos mayores que, sin embargo, son capaces de prevenir delitos mediante estrategias de observación y seguimiento de casos para contrarrestar la opacidad e impunidad de los delitos cometidos en localidades (Mejía, Carreón y García, 2016).

Sin embargo, García et al., (2016) advierten que los delitos no sólo se han trasladado de los espacios públicos a los ámbitos íntimos digitales tales como; las redes de Facebook, Instagram, WhatsApp, Twitter o Periscope, sino además se han desarrollado identidades violentas como los stalkers o los bullers.

Incluso, las nuevas formas de inseguridad y violencia coexisten con las desapariciones forzadas, secuestros y genocidios perpetrados por el Estado como es el caso de los 43 estudiantes del municipio de Ayotzinapa, cabecera de Iguala en el estado de Guerrero (centro de México), ya que la propaganda del gobierno incidió en las percepciones de inseguridad de ciudadanía al difundir una "verdad histórica" que consiste en legitimar la rectoría del Estado como único actor capaz de explicar el crimen de lesa humanidad (García, Sandoval y Aguilar, 2016).

Por lo tanto, la diversidad, pluralidad y heterogeneidad de las propagandas supone múltiples identidades civiles que es posible estudiar en un modelo integral, observar sus similitudes y diferencias, así como anticipar escenarios que van del genocidio hasta la indiferencia de sectores de la sociedad civil. 


\section{Referencias}

Acosta, J. (2010). Violencia política y derechos humanos. Universita ria. $11,1-13$

Añez, M., Rujano, R. y Párraga, J. (2011). Seguridad ciudadana y acceso a la justicia. Cuestiones J uńd ic as. 5, 11-25

Bizer, G., Larsen, J. y Petty, R. (2010). Exploringthevalenceframingeffect: negativeframingenhancesattitudestrength. Po litic a IPsychology. 32, 59-80 Borghello, C. y Temperi, M. (2012). Suplantando la identidad digital como delito informático. Revista de Info mátic a y Derecho. 41, 78-93

Calderón, E. (2012). La impronta legal de la participación de las fuerzas armadas en seguridad pública: el caso de la lucha contra el narcotráfico en América del Sur. Revista Latinoamericana de Seguridad Ciudadana. 12, 97-109

Campillo, C. (2012). La gestión estratégica de la información municipal. Análisis de temas, su tratamiento e irrupción en el ayuntamiento del Elche (1995-2007). Revista de Estrategia, tendencia e Innovación de la Comunicación. 3, 149170

Carcelén, R., Esteba, P. y Peyró, L. (2013). Tratamiento informativo de las drogas en medios de salud en España y su relación con la agenda científica. Revista Iberoamericana para la Investigación y el Desarrollo Educ a tivo, 10, 1-35

Carreón, J. (2013). Hacia la construcción de una esfera civil de seguridad e identidad pública. Eleuthera, 9 (2), 95-115

Carreón, J. (2014). Emprendedurismo migrante y comerciante. Tlatemoani, 15, 158-187

Chávez, J., Carreón, J., Hernández, J. y García, C. (2016). Análisis factorial confirmatorio con mínimos cuadrados ordinarios no 
ponderados de una escala de actitudes hacia el duelo. Revista Psic umex, 6(1), 24-38

Flores, L. y Mendieta, A. (2012). La percepción de la nota roja periodística en primera plana, un estudio de caso. Revista de Comunicación. 14, 1-13

Fondevilla, G. y Quintana, M. (2013). Juego de palabras: los discursos presidenciales sobre el crimen. Estud ios Soc iológic os,31 (93), 721-754

García, C. (2010). La estructura de la actitud de atención tanatológica. Funda mentos en Humanidades, 11 (1), 121-132

García, C. (2011). Estructura de la inseguridad pública. Liberabit. 18, 3744

García, C. (2012). Sistemas racionales sociopsicológicos. Diálogos de Derecho y Política, 11 (4), 1-14

García, C. (2013). Sistemas de violencia sociopolítica. Polis, 12 (36), 243265

García, C. (2014). La formación del capital humano en la civilización del cambio climático. Revista Intema c ional de Ciencias Social, 10, 107-125

García, C., Hernández, J., Aguilar, J., Morales, M. y Peralta, M. (2016). Confiabilidad y validez de un instrumento que mide determinantes perceptuales del acoso a través de Internet. Revista Encuentros, 14 (1), 103-117 [DOI: 10.15665/re.v14i1.672 ]

García, C., Sandoval, J. y Aguilar, J. (2016). Aproximación al encuadre de los medios impresos en torno a la atención a víctimas del caso Ayotzinapa, Iguala, Guerrero (México). Revista de Comunicación del SEC CI, 20 (39), 153-160

González, C., Pombo, O., Méndez, H., Espejel, M. y Leyva, J. (2011). Representaciones mediáticas socioambientales. Revista Culturales. 13, 145-172

González, J., Hernández, A. y Garza, R. (2010). Modelo predictivo de las interacciones violentas en parejas jóvenes y prejuicio de género asociados. Revista Científica de Psicología. 10, 224-239

Groshek, J. (2011). Media, instability, a democracy: examiningthegranger causal relationships of the 122 countriesfrom 19432003. J oumal of Communic ation. 61, 1161-1182

Gu, M. y Goldfarb, B. (2010). Affect and theframingeffectwittingindividualsover time: risktaking in a 
dynamicinvestmentsimulation. Academic of Management Joumal. 53, $411-431$

Guardiola, A., Espinar, E. y Hernández, I. (2010). Los inmigrantes como amenaza en la televisión española. Convergencia, 53, 59-58

Humanes, M. y Moreno, M. (2012). El efecto agenda sobre los temas de campaña en las elecciones generales de 2008. Revista de Estrategia, Tendencia e Innovación de la Comunica ción. 3, 191-207

Izquierdo, L. (2012). La uniformidad temática en las secciones de internacional de los diarios madrileños frente a las secciones locales. Communic ationPapers, media Literacy\&GenderStudies. 1, 97-104

Latorre, E. (2011). Visibilización de la memoria de las víctimas de la violencia en el departamento de Magdalena: Resiliencia para construir verdad jurídica. Prolegomenos, Derechos y Valores, 27, 199-212

Mao, Y., Richter, M., Burns, K. y Chaw, J. (2012). Homelessnesscoverage, social reality, and media ownerships: comparingsomenationalnewspaperswith to regional newspapers in Canada. MassC ommunication\&J ouma lism. 2, 1-7

Mejía, S., Carreón, J. y García, C. (2016). Efectos psicológicos de la violencia e inseguridad en adultos mayores. Revista Eureka, 13 (1), 39-55

Navarro, Y., Climent, J. y Fernández, J. (2012). Modelos de gestión de conflictos en serie de ficción televisiva. Escritos de Psic ología , 5, 52-60

Nisbet, E., Stoycheeff, E. y Pearce, K. (2012). Internet use and democraticdemands: a multinational, multilevelmodel of Internet use and citizenattitudesaboutdemocracy. Joumal of Communication, 62, 249-265

Orozco, G. y Franco, D. (2012). Las audiencias convergentes y su investigación: análisis de recepción transmedial de la serie El Equipo. Derecho a Comunicar, 5, 46-63

Rodríguez, F. (2010). Discurso xenófobo y fijación de agenda. Un estudio de caso en la prensa de Canarias (España). Revista Latina de Comunic a ción Social, 65, 222-230

Salas, L. (2011). La seguridad pública y su apoyo en políticas públicas. Encrucija da Revista de Estudios en Administración Pública . 8, 1-12

Ulloa, I. (2012). El desafío de garantizar la seguridad pública: el modelo de seguridad pública. Seguridad y Ciudadanía. 7, 13-24 
Wasike, B. (2013). Framingnews in 140 characters: how social media editorsframethenews and interactwithaudiencesvia twitter. Global Media J oumal, 6, 5-23

Wirth, W., Matthes, J., Schemer, C., Wettstein, M., Friemel, T., Hânggli, R. y Siegert, G. (2010). Agenda building and setting in referendumcampaign: investigatingtheflow of argumentsamongcampaigners, the media, and thepublic. J ouma lism\&Mass Media Communic ation. 87, 328-345

Zaffaroni, R. (2011). Estado y seguridad pública: algunas consideraciones básicas. Cua demos de Seguridad. 14, 17-32

Zúñiga, L. (2012). Desafíos institucionales de la colaboración policialmilitar el triángulo norte. Revista Latinoamericana de Seguridad Ciudadana. 12, 83.96 